\title{
LA PROMOCIÓN DEL EMPRENDIMIENTO SOCIAL MEDIANTE METODOLOGÍAS INNOVADORAS: HACIA UN NUEVO PARADIGMA EDUCATIVO
}

\author{
The Promotion of Social Entrepreneurship by Means of Innovative \\ Methodologies: Towards a New Educational Paradigm
}

Josune López RodRÍGuez

Universidad de Deusto (Bilbao)

\section{ABSTRACT}

En la actualidad, el mundo está inmerso en una grave crisis económica, social y medioambiental que demanda nuevas acciones para garantizar un futuro sostenible y socialmente justo. Ante este desafio, una de las posibles respuestas consiste en promover el emprendimiento social porque este fenómeno pretende abordar las necesidades sociales y contribuir al desarrollo social y económico. A tal fin, como paso previo, conviene crear una conciencia social sobre su alcance, para lo cual el ámbito educativo puede ser un contexto apropiado. En esta labor, más allá de las metodologías expositivas, es indispensable implantar metodologias activas e innovadoras que estimulen el espiritu crítico y emprendedor de los ciudadanos. Teniendo en consideración estas cuestiones, el presente artículo tiene por objeto verificar, desde una perspectiva teórica, la conveniencia de emplear metodologías innovadoras, como el aprendizajeservicio, el aprendizaje por proyectos, el aprendizaje basado en problemas y el aprendizaje cooperativo, para promover el emprendimiento social desde la educación.

Palabras clave: Emprendimiento social, metodologias innovadoras, innovación educativa. 
Gaur egun, mundua ekonomia, gizarte eta ingurugiro krisi sakon batean murgilduta dago. Egoera honi aurre egiteko eta etorkizun justu eta iraunkor bat bermatzeko, beharrezkoak dira egintza berriak. Hain zuzen, gizarte-ekintzailetza bultzatzea erantzun posible bat da, izan ere, fenomeno honek helburutzat du gizarte-beharrei aurre egitea eta gizarte eta ekonomia garapena bultzatzea. Xede hau lortzeko, eta aldez aurreko urrats moduan, fenomeno honen norainokoaren inguruan gizarte kontzientzia bat sortzea komeni da eta, horretarako, hezkuntza arloa testuinguru aproposa izan daiteke. Zeregin honetan, azalpenezko metodologiez gain, metodologia aktibo eta berritzaileak erabiltzea nabitaezkoa da hiritarren espiritu kritikoa eta ekintzailea suspertzeko. Premisa hauek kontuan izanik, artikulu honek helburutzat du, ikuspuntu teoriko batetik behintzat, metodologia berritzaileen erabilpenaren egokitasuna egiaztatzea, hala nola, ikasketa-zerbitzua, proiektu bidezko ikasketa, arazoan oinarritutako ikasketa eta ikasketa kooperatiboa, gizarte-ekintzailetza bultzatzeko.

Hitz gakoak: Gizarte-ekintzailetza, metodologia berritzaileak, hezkuntza berritzailea.

Today, the world is immersed in a serious economic, social and environmental crisis that requires new actions in order to ensure a sustainable and socially fair future. In face of this challenge, a possible response might be the promotion of social entrepreneurship as this phenomenon pretends to address social needs and contribute to social and economic development. To that end, and as a previous step, it is convenient to foster social awareness on its scope, for which the educational field can be an appropriate context. In this regard, beyond the expositive methodologies, it is essential to implement active and innovative methodologies that encourage the critical and entrepreneurial spirit of citizens. Taking these premises into consideration, this paper aims to verify, from a theoretical perspective, the convenience of implementing innovative methodologies, such as, service-learning, project based learning, problembased learning and cooperative learning, in order to promote social entrepreneurship from education.

Keywords: Social entrepreneurship, innovative methodologies, innovative education. 


\section{SUMARIO}

Sumario. 1. Introducción. 2. Marco teórico. 2.1. Emprendimiento social. 2.2. Metodologías innovadoras. 2.2.1. Aprendizaje-servicio. 2.2.2. Aprendizaje por proyectos. 2.2.3. Aprendizaje basado en problemas. 2.2.4. Aprendizaje cooperativo. 3. Discusión. 4. Conclusiones. 5. Bibliografía.

\section{Introducción}

El actual escenario mundial, caracterizado por una profunda crisis económica, social y medioambiental, está siendo testigo de constantes y sistemáticas violaciones de los derechos humanos. Esta alarmante situación exige nuevas respuestas y nuevos horizontes de actuación para avanzar hacia un futuro sostenible y socialmente justo.

En este contexto de incertidumbre, una de las posibles medidas a adoptar consiste en promover el emprendimiento social, en el marco de una Economía Social, por ser éste un fenómeno que tiene por objeto afrontar las necesidades sociales y contribuir al desarrollo social y económico. Precisamente, en los últimos años, distintas organizaciones internacionales y regionales han destacado el relevante papel del emprendimiento social en la lucha contra la pobreza y los problemas sociales y han remarcado, al mismo tiempo, su trascendencia como mecanismo de progreso social e inserción sociolaboral.

Ahora bien, tal y como afirma Batlle, "no se nace emprendedor social», sino que se aprende a serlo (Batlle, 2012: 2). En consecuencia, resulta indispensable construir una conciencia social acerca del significado y alcance de este fenómeno. Concretamente, la comunidad educativa, por su importancia en la formación integral de las personas, puede constituir un medio idóneo para transmitir y erigir las bases que componen el emprendimiento social. En la consecución de este cometido, el mero aprendizaje de conceptos teóricos sobre el emprendimiento social no resulta suficiente y, por eso, es necesario buscar alternativas a los métodos tradicionales de enseñanza (Pérez, 2011: 57).

En base a estas premisas, la hipótesis del presente trabajo radica en el hecho de que el empleo de metodologías activas e innovadoras como el aprendizajeservicio, el aprendizaje por proyectos, el aprendizaje basado en problemas y el aprendizaje cooperativo constituye una herramienta apropiada para transmitir 
y trabajar los contenidos, los valores y las competencias que caracterizan al emprendimiento social. Partiendo de esta hipótesis, el objetivo de este artículo consiste en valorar, desde una perspectiva teórica, la idoneidad de la implantación de metodologías innovadoras como mecanismo para promover el emprendimiento social desde el ámbito educativo.

Con el fin de lograr el objetivo descrito, el presente trabajo se estructura de la siguiente forma: primero, se expone el marco teórico en el que se analiza el significado del emprendimiento social y de las metodologías innovadoras; partiendo de este marco teórico, se aborda la discusión sobre el empleo de las metodologías innovadoras como herramienta para promover el emprendimiento social; y, por último, se exponen las conclusiones más importantes del trabajo.

Para lograr el objetivo propuesto se han empleado, esencialmente, los métodos descriptivo, comparativo y exploratorio.

\section{Marco teórico}

Para poder examinar y valorar el empleo de metodologías innovadoras como estrategia para aprender a emprender socialmente, primero, resulta conveniente estudiar, por separado, el alcance del emprendimiento social y de las metodologías innovadoras.

\subsection{Emprendimiento social}

En el momento presente no hay una definición única y consensuada de la expresión «emprendimiento social», sino que coexisten numerosas definiciones.

A grandes rasgos, Ashoka, la mayor red internacional de emprendedores sociales, concibe el emprendimiento social como un pilar fundamental para promover un desarrollo sostenible. Esta organización no define el alcance del fenómeno, sino que se refiere a los «emprendedores sociales», definiéndolos como "los individuos que formulan y presentan nuevas soluciones ante cuestiones sociales» (Ashoka, 2016: 6) ${ }^{1}$.

Por su parte, Mair y Martí definen el emprendimiento social como «un proceso que integra la utilización y combinación innovadora de recursos orientados a la búsqueda de oportunidades en aras a favorecer un cambio social y/o abordar necesidades sociales» (Mair y Martí, 2006: 37)2. En concreto, estos autores conciben el emprendimiento social como un proceso; es decir, como una práctica evolutiva conformada por distintas etapas de exploración, formación, creación, cre-

\footnotetext{
1 Traducción propia.

2 Traducción propia.
} 
cimiento, desarrollo y fortalecimiento (Moreira y Urriolagoitia, 2011: 28). Según los autores, se trata de un proceso de creación de valor basado en la combinación innovadora de recursos. A través de esta aleación de recursos, se pretende explorar, analizar y explotar oportunidades con el fin de crear valor social estimulando, de este modo, el cambio social o haciendo frente a las necesidades sociales.

Otros autores, como Austin, Stevenson y Wei-Skillern, se refieren al emprendimiento social como "una actividad innovadora de creación de valor social que puede darse en el sector no lucrativo, de negocios o gubernamentali (Austin et al., 2006: 2) ${ }^{3}$. Así, estos autores consideran que el principal propósito del emprendimiento social es la creación de valor social para el bien común.

Combinando las perspectivas teórica y práctica, Roberts y Woods definen el emprendimiento social como "la construcción, evaluación y búsqueda de oportunidades para el cambio social transformador, llevado a cabo por individuos visionarios, apasionados y entregados» (Roberts y Woods, 2005: 49)4. Más concretamente, los autores explican que el emprendimiento social «es un constructo que cubre un importante vacio entre los negocios y la benevolencia», esto es, «supone la aplicación del emprendimiento en la esfera social» (Roberts y Woods, 2005: 45).

Los autores Martin y Osberg precisan que el emprendimiento social es un fenómeno que engloba tres elementos principales (Martin y Osberg, 2007: 35). En primer lugar, la identificación de un equilibrio estable pero injusto que causa exclusión, marginalización o sufrimiento a una parte de la humanidad que carece de medios financieros o de influencia política para conseguir beneficios transformadores por cuenta propia; en segundo lugar, la identificación de una oportunidad en este equilibrio injusto, promoviendo una propuesta de valor social y haciendo uso de la inspiración, la creatividad, la acción directa, el coraje y la fortaleza, desafiando, de esta forma, la estable hegemonía estatal. Y, en tercer lugar, la proyección de un nuevo y estable equilibrio que lanza el potencial atrapado o alivia el sufrimiento del grupo social en el que se concentre, y que mediante la imitación y la creación de un ecosistema duradero alrededor del nuevo equilibrio garantiza un futuro mejor para dicho grupo o, incluso, para la sociedad en su conjunto5.

En el contexto español, siguiendo la línea de Martin y Osberg, Guil y Montoya describen el emprendimiento social como un fenómeno que "procura valor social sostenible [...] a través del desarrollo de oportunidades para el cambio social [...], en aquellos sectores en donde se identifica un equilibrio estable pero injusto que causa exclusión, marginalización o sufrimiento a determinados segmentos de la

\footnotetext{
3 Traducción propia.

4 Traducción propia.

5 Traducción propia.
} 
población» (Guil y Montoya, 2015: 182). En esta definición se incide especialmente en la creación y desarrollo de oportunidades para lograr un cambio social en determinados sectores.

En base a los conceptos expuestos, y al hilo de la idea plasmada al inicio del apartado, debe afirmarse que no existe actualmente una definición única sobre el emprendimiento social. Debe apuntarse, además, que el alcance del emprendimiento social dependerá del contexto en el que se enmarque y que, en consecuencia, estará condicionado por las circunstancias sociales, políticas, culturales y económicas que lo rodeen (Austin et al., 2006: 5; Mair y Martí, 2006: 42, Moreira y Urriolagoitia, 2011: 29).

No obstante estas cuestiones, puede identificarse en todas las definiciones un elemento común que caracteriza al emprendimiento social, a saber, la búsqueda de soluciones a los problemas y las necesidades sociales (Moreira y Urriolagoitia, 2011: 20). Ciertamente, el emprendimiento social implica la detección e identificación de problemas y necesidades sociales, en los que encuentra un espacio de oportunidad para conseguir un cambio social. Así, el emprendimiento social persigue de forma prioritaria un fin social, esto es, pretende lograr un beneficio para la sociedad, ello sin perjuicio de que también pueda procurar un rendimiento económico (Mair y Martí, 2006: 39).

En base a estas características, los emprendedores sociales desarrollan una serie de competencias que se convierten en "verdaderos valores éticos», a saber: la capacidad para detectar e identificar la existencia de una desigualdad, la búsqueda de una justicia social y la toma de decisiones desde una posición empática (Blanco et al., 2012: 50).

\subsection{Metodologías innovadoras}

Durante las últimas décadas, el modelo educativo está siendo objeto de una patente transformación. Efectivamente, se está produciendo una evolución desde un modelo tradicional basado en la enseñanza hacia un modelo fundamentado en el aprendizaje (Fernández, 2006: 36). Estos cambios están reduciendo significativamente el protagonismo de los docentes y están situando a los discentes en el centro del proceso de enseñanza-aprendizaje. Este nuevo contexto educativo exige complementar el sistema tradicional de transmisión de conocimientos con nuevas metodologías y técnicas que fomenten la participación y autonomía de los estudiantes (Pérez, 2011: 57).

A este respecto, debe señalarse que no hay una única metodología para conseguir dicho fin (Pérez, 2011: 58). Al contrario, actualmente coexisten numerosas metodologías que, entre otras funciones, fomentan el protagonismo del alumnado. Por eso, la elección de una metodología concreta o la combinación de diversas metodologías dependerá, caso por caso, de distintos factores como 
los objetivos perseguidos, el contexto, el número de alumnos, los recursos o el tiempo disponible.

Teniendo presente el propósito de este trabajo, a continuación se realizará un breve análisis del alcance de cuatro metodologías innovadoras por su especial vinculación con la promoción del emprendimiento social, esto es, el aprendizaje-servicio, el aprendizaje por proyectos, el aprendizaje basado en problemas y el aprendizaje cooperativo, ello sin perjuicio de que existan otras metodologías apropiadas para lograr esta finalidad. Cabe matizar que no se trata de metodologías nuevas, ya que, siquiera bajo otras denominaciones y en contextos diferentes, estas metodologías cuentan con antecedentes en épocas anteriores. Ello no obstante, las mismas están cobrando cada vez más importancia en el seno de la comunidad educativa y están adaptándose a las demandas del mundo actual, de ahí que sean consideradas metodologías innovadoras.

\subsubsection{Aprendizaje-servicio}

En el contexto educativo español, la definición del aprendizaje-servicio por excelencia es la elaborada por Puig, Batlle, Bosch y Palos, quienes lo han definido como "una metodología que combina procesos de aprendizaje y de servicio a la comunidad en un solo proyecto bien articulado en el que los participantes se forman al trabajar sobre necesidades reales del entorno con el objetivo de mejorarlo» (Puig et al., 2007: 20 y Puig, 2011: 11).

En términos generales, este concepto hace alusión a dos elementos principales, a saber, el proceso de aprendizaje y el servicio a la comunidad, situándolos al mismo nivel y considerándolos como elementos complementarios e inseparables. Además, estos elementos deben estar correctamente articulados en un proyecto educativo (Lucas y Martínez, 2012: 6 y Santos et al., 2015: 20). Precisamente, el aprendizaje-servicio requiere una correcta planificación y programación de las actividades de aprendizaje y servicio que van a desarrollarse, así como la delimitación de un sistema de evaluación. Más aún, el aprendizaje-servicio exige el desarrollo de una reflexión constante para valorar su eficacia y considerar posibles cambios (Uruñuela, 2011: 40).

\subsubsection{Aprendizaje por proyectos}

A través del aprendizaje por proyectos, los alumnos parten de un problema o de una necesidad y, en base a los mismos, definen su propósito, que debe consistir en crear un producto final. Para alcanzar este fin, los estudiantes deben realizar un estudio de mercado, crear un plan para gestionar el proyecto y diseñar y elaborar el producto (Rodríguez-Sandoval et al., 2010: 16). El elemento que caracteriza a esta metodología es el producto final que constituye, en esencia, la culminación del proyecto. Así, en la preparación, indagación y desarro- 
llo del proyecto los alumnos trabajan de forma autónoma y cooperativa, manteniendo una estrecha conexión con la realidad y los problemas diarios (De la Calle, 2016: 8).

\subsubsection{Aprendizaje basado en problemas}

La metodología del aprendizaje basado en problemas conlleva la preparación por parte de los docentes de una recopilación de problemas, descritos de forma clara y sencilla, que son presentados al alumnado (Vizcarro y Juárez, 2008: 19). Así, los alumnos deben realizar un análisis crítico y estructurado del problema planteado en aras a buscar una explicación y resolverlo. A tal fin, los estudiantes deben identificar las cuestiones en las que profundizar, plantear distintas hipótesis, detectar posibles lagunas o vacíos de conocimiento, discutir sobre la información recopilada y, en base al trabajo realizado, deben resolver el problema e, incluso, poner sobre la mesa nuevos problemas detectados (Manzanares, 2008: 22-23 y Grimes, 2015: 364).

El componente central de esta metodología es el problema que se plantea al alumnado (Restrepo, 2005: 11). En consecuencia, la elección, preparación y descripción del mismo constituyen un factor fundamental para el éxito de la metodología. En esta labor, resulta esencial escoger problemas de actualidad, del mundo real, para lograr despertar el interés de los alumnos e incrementar su motivación (Romero y García, 2008: 37 y 42-43). Si bien esta metodología guarda cierta similitud con el aprendizaje basado en proyectos, la principal diferencia entre ambas radica en que, mientras en el aprendizaje basado en proyectos se otorga una especial importancia al producto final, en el aprendizaje basado en problemas se valora fundamentalmente el proceso que lleva a los alumnos a resolver el problema planteado (Del Moral y Sobrino, 2016: 5).

\subsubsection{Aprendizaje cooperativo}

El aprendizaje cooperativo implica la organización de los alumnos en equipos reducidos y heterogéneos para llevar a cabo una tarea concreta. Los componentes del equipo tienen un fin común y deben cooperar de forma activa y directa para conseguirlo. En el marco del aprendizaje cooperativo, los integrantes del equipo no solamente pretenden obtener un beneficio personal con su trabajo sino que también procuran un beneficio para el resto de los miembros (Johnson et al., 1994: 5, Prieto, 2007: 32 y Sharan, 2014: 802).

Según Johnson, Johnson y Holubec el aprendizaje cooperativo se compone de cinco elementos básicos (Johnson et al., 1994: 9-10). El primer elemento fundamental de esta metodología es la interdependencia positiva. Es decir, el equipo de trabajo tiene que tener asignado un cometido claro y un objetivo concreto, de tal forma que los miembros asuman que es indispensable la participación y contribución de todos para alcanzar el propósito planteado. El se- 
gundo componente es la responsabilidad, tanto individual como grupal. Ciertamente, el equipo en su conjunto debe comprometerse a conseguir el objetivo delimitado y, al mismo tiempo, cada integrante debe asumir la responsabilidad de cumplir con las tareas que le corresponden. El tercer elemento consiste en la interacción estimuladora, en virtud de la cual cada uno de los integrantes del equipo debe promover e incentivar el éxito del resto de los miembros. El cuarto elemento implica el aprendizaje y desarrollo de técnicas interpersonales y grupales. A través de estas técnicas, los alumnos asimilan cómo ejercer labores de dirección, cómo tomar decisiones, cómo comunicarse y cómo gestionar los conflictos. Y el último componente esencial es la evaluación. En efecto, resulta imprescindible que el equipo realice una evaluación constante del trabajo realizado y se lleven a cabo las modificaciones necesarias para conseguir de forma eficaz los objetivos propuestos.

\section{Discusión}

En la actualidad, el emprendimiento social, en el contexto de una Economía Social, se presenta como un mecanismo esperanzador para atender las necesidades sociales, afrontar los problemas arraigados en la sociedad y recuperar el bien común (Marin et al., 2012: 150 y Bravo Monge, 2016: 26). Ciertamente, el fracaso de la actuación de las instituciones públicas, los gobiernos, las entidades sin ánimo de lucro y las empresas capitalistas en la atención y solución de los problemas sociales, así como los recientes cambios demográficos, la liberalización económica, la flexibilidad del mercado laboral y los progresos en la tecnología, han favorecido e impulsado la aparición y desarrollo del emprendimiento social (Moreira y Urriolagoitia, 2011: 19 y Zahra et al., 2009: 520).

En el plano internacional, la Organización para la Cooperación y el Desarrollo Económicos determinó en el Resumen Ejecutivo de los informes de base de la II Conferencia de Ministros encargados de pequeñas y medianas empresas, del año 2004, que los países son cada vez más conscientes de que el espíritu emprendedor social contribuye a reducir la pobreza y a dar voz a grupos sociales especialmente vulnerables. En dicho informe, este organismo de cooperación internacional destacó el importante papel de la educación y la formación en la promoción de la cultura emprendedora, animando a los países a introducir el espíritu emprendedor en todos los niveles educativos (OCDE, 2004: 7).

Por su parte, la Unión Europea viene manifestando en los últimos años la necesidad de afrontar los nuevos desafíos mundiales y la cambiante situación económica con la participación de ciudadanos innovadores y emprendedores (Comisión Europea, 2014: 7 y Eurydice, 2012: 5). En concreto, la Comisión 
Europea declaró en la Comunicación relativa a Un nuevo concepto de educación: invertir en las competencias para lograr mejores resultados socioeconómicos ${ }^{6}$, que la educación para el emprendimiento resulta fundamental. En esta línea, en la Comunicación relativa al Plan de acción sobre emprendimiento 2020 ${ }^{7}$, del año 2013, la Comisión propuso tres áreas de intervención para promover el emprendimiento en Europa, entre las cuales incluyó la necesidad de educar y formar en materia de emprendimiento.

En octubre de 2016, el Grupo de expertos de la Comisión Europea sobre el emprendimiento social publicó un informe sobre las empresas sociales y la Economía Social en el que explicaba que el emprendimiento social debería integrarse de forma más efectiva en los currículos educativos (Comisión Europea, 2016: 22). En dicho informe, el Grupo de expertos insta a los Estados Miembros a promover una cultura sobre el emprendimiento social para lo cual les sugiere destinar más financiación a universidades, escuelas de enseñanza media, organizaciones de educación informal y programas educativos centrados en la empresa social, Economía Social y emprendimiento social (Comisión Europea, 2016: 23).

Estas declaraciones ponen de manifiesto el interés de las instancias internacionales y europeas por el fomento de la Economía Social, las empresas sociales y el emprendimiento social. Más aún, se percibe una clara tendencia a apoyar la promoción del espíritu emprendedor social desde el ámbito educativo, una postura que ha sido avalada por la doctrina científica y por distintas organizaciones sociales.

Desde Ashoka, Gallego sostiene que los emprendedores sociales son indispensables para el progreso y la evolución de las sociedades (Gallego, 2011: 155).

Al hilo de esta idea, Melián y Campos defienden la promoción del espíritu emprendedor y su canalización hacia empresas de Economía Social como mecanismo para afrontar el actual contexto de crisis, al tiempo que subrayan la necesidad de incrementar el conocimiento acerca de este sector (Melián y Campos, 2010: 63-64).

Asimismo, Blanco, Garín y Rodríguez declaran que, en la actualidad, la capacidad de iniciativa y la creatividad constituyen «valores fundamentales para el desarrollo de los individuos y para el progreso y crecimiento de la sociedad en su conjunto» (Blanco et al., 2012: 45).

A este respecto, Del Real, Bueno y Fernández resaltan el importante papel del sistema educativo como primer contacto de la ciudadanía con los valores propios del emprendimiento y defienden que es necesario promover el empren-

\footnotetext{
$6 \operatorname{COM}(2012) 669$ final, de 20 de noviembre de 2012

7 COM(2012) 795 final, de 9 de enero de 2013.
} 
dimiento desde las instituciones educativas para lograr un cambio social (Del Real et al., 2012: 102).

Al hilo de esta idea, Batlle defiende que deben replantearse las estrategias educativas para fomentar el emprendimiento social desde la educación. En concreto, la autora señala que es indispensable desarrollar y fomentar un entrenamiento progresivo hacia el emprendimiento social (Batlle, 2012: 1).

Según Pérez, la sociedad actual exige ciudadanos responsables con capacidades "que van más allá del mero conocimiento", lo que implica la necesidad de desarrollar la capacidad de aprender y emprender mediante métodos que fomenten la participación del alumnado en el proceso de enseñanza-aprendizaje (Pérez, 2011: 57).

Igualmente, la Fundación Príncipe de Girona destaca el papel de la educación en la promoción del emprendimiento y, más concretamente, en el fomento del emprendimiento social. Así, se determina que los proyectos educativos de emprendimiento que envuelven un compromiso ético con una sociedad justa no solamente implican el desarrollo por parte del alumnado de la autonomía y la iniciativa propia sino que también suponen una inversión social para garantizar un futuro socialmente responsable (Fundación Príncipe de Girona, 2013: 56). En lo que respecta al desarrollo de la competencia relativa a aprender a emprender, desde la Fundación se defiende que es imprescindible el empleo sistemático de metodologías activas y, a este respecto, se sostiene que el aprendizaje-servicio, el aprendizaje por proyectos, el aprendizaje basado en problemas y el aprendizaje cooperativo constituyen metodologías eficaces para lograr dicha finalidad. Además, se reconoce que las iniciativas que tienen una mayor repercusión en el aprendizaje del alumnado son aquellas que mantienen un vínculo con hechos, situaciones y retos reales porque, además de fomentar la iniciativa, autonomía y creatividad de los estudiantes, les ayudan a comprender la complejidad de la sociedad en la que viven, a detectar las necesidades sociales y a actuar para conseguir una transformación social (Fundación Príncipe de Girona, 2013: 49-50).

En consonancia con este planteamiento, numerosos autores han secundado que el aprendizaje-servicio, el aprendizaje por proyectos, el aprendizaje basado en problemas y el aprendizaje cooperativo constituyen metodologías motivadoras que fomentan la participación de los discentes y pueden constituir, además, una herramienta apropiada para que el alumnado desarrolle las características básicas de los emprendedores sociales, como la autonomía, la iniciativa, la creatividad, la responsabilidad social, el liderazgo, el espíritu crítico, la preocupación por los problemas sociales, la búsqueda de soluciones, el trabajo en equipo, la empatía, la asunción de riesgos o los valores cooperativos (entre otros, De la Calle, 2016: 8; Marín et al., 2012: 154; Puig et al., 2007: 212, Vizcarro y Juárez, 2008: 34). 
En base a las posturas expuestas, parece que hay un cierto consenso en la doctrina acerca de la necesidad de fomentar el emprendimiento social desde el ámbito educativo y acerca de los beneficios que puede reportar la introducción de metodologías innovadoras y activas para desarrollar las facultades propias de los emprendedores sociales. Así, en principio, las iniciativas de aprendizaje-servicio, aprendizaje por proyectos y aprendizaje basado en problemas pueden llegar a comprender los elementos propios de un proyecto de emprendimiento social. A través de estas metodologías, los alumnos pueden desarrollar sus capacidades para comprender la realidad social, detectar necesidades y problemas sociales, identificar espacios de oportunidad, proponer ideas, aportar soluciones y trabajar la creatividad (Diego, 2015: 22). Asimismo, mediante el aprendizaje cooperativo, el alumnado interioriza el valor de la diversidad, la responsabilidad, la solidaridad y el compromiso (Domingo, 2008: 233 y Prieto, 2007: 109). Más aún, el aprendizaje cooperativo permite el desarrollo de la habilidad de ponerse en el lugar de otra persona, de comprender sus inquietudes y de empatizar con ella, al tiempo que fomenta la capacidad para detectar y resolver problemas (Vinuesa, 2002: 138-139).

Ello no obstante, existen determinados factores que pueden poner en entredicho la eficacia de este planteamiento y que, por consiguiente, deben tenerse en consideración.

A este respecto, desde la Fundación Príncipe de Girona se señala que las actividades y experiencias promovidas desde el ámbito educativo relacionadas con el emprendimiento únicamente conllevarán un aprendizaje significativo si van acompañadas de "un tratamiento didáctico adecuado en la metodología, la reflexión y el seguimiento en el tiempo" (Fundación Príncipe de Girona, 2013: 49).

Por su parte, Marina advierte que, en muchas ocasiones, las iniciativas didácticas orientadas a desarrollar la competencia de emprender se centran, sobre todo, en el aspecto empresarial y económico del emprendimiento y olvidan los propósitos personales y sociales del mismo. Ante esta realidad, el autor sugiere que las iniciativas didácticas para promover el emprendimiento se enmarquen dentro de un proyecto ético (Marina, 2010: 60 y 69).

Junto con estas observaciones, y en relación con las metodologías innovadoras a las que se ha hecho alusión, la doctrina pone de manifiesto una serie de causas que pueden dificultar y limitar su efectividad.

Así, algunos autores señalan que es muy común la existencia de cierta inseguridad e incertidumbre ante los cambios metodológicos e, incluso, la presencia de reticencias al respecto (Del Valle y Villa, 2008: 145). En relación con estas sensaciones iniciales, los autores inciden en la necesidad de asumir responsabilidades, tomar las cautelas necesarias y llevar a cabo acciones apropiadas para conseguir los objetivos propuestos, haciendo especial hincapié en la importancia de 
planificar las estrategias metodológicas desde una perspectiva realista, esto es, teniendo en consideración el contexto, los recursos con los que se cuenta, el grado de madurez de los destinatarios y el tiempo disponible (Battle, 2013: 134-135).

Al hilo de este último apunte, el tiempo es considerado como uno de los principales obstáculos para el empleo de metodologías innovadoras. En efecto, la aplicación exitosa de metodologías en las que los alumnos se convierten en agentes activos de su aprendizaje requiere un amplio margen de tiempo para su preparación, ejecución y evaluación (Del Valle y Villa, 2008: 145 y Vizcarro y Juárez, 2008: 34).

Asimismo, tal y como se acaba de apuntar, la utilización de estas metodologías exige un alto grado de implicación, participación y compromiso por parte del alumnado. Como consecuencia, algunos alumnos pueden experimentar situaciones de ansiedad, estrés, frustración o incomodidad (Deeley, 2016: 97 y Del Valle y Villa, 2008: 143).

Otro de los obstáculos que destacan los autores es la falta de preparación y formación del profesorado en torno a estas metodologías, lo que genera un clima de desconocimiento e incertidumbre sobre su aplicación (Salinas y Osorio, 2012: 149).

\section{Conclusiones}

A lo largo del trabajo se ha pretendido probar la autenticidad de la hipótesis planteada en la introducción y alcanzar el objetivo propuesto.

En base al estudio realizado, cabe concluir que el emprendimiento social, enmarcado en el contexto de la Economía Social, adquiere una especial relevancia ante la preocupante realidad socioeconómica, política y medioambiental actual. Para promover y consolidar este tipo de emprendimiento es necesario, como paso preliminar, crear una conciencia social sobre su alcance y repercusión. A partir del análisis precedente, se deduce que el ámbito educativo es un espacio apropiado para despertar en los ciudadanos el interés por los problemas y las necesidades sociales. A este respecto, se ha comprobado que resulta imprescindible ir más allá de la simple transmisión de conocimientos y desarrollar estrategias innovadoras que estimulen la participación activa del alumnado. Concretamente, el estudio de las metodologías relativas al aprendizaje-servicio, el aprendizaje por proyectos, el aprendizaje basado en problemas y el aprendizaje cooperativo demuestra que estos métodos pueden constituir un entrenamiento eficaz de las destrezas y capacidades vinculadas al emprendimiento social.

Estas conclusiones se fundamentan, sin embargo, en un análisis meramente teórico. En este sentido, la ausencia de una fundamentación práctica implica 
que no se pueda corroborar su eficacia real. Esta limitación evidencia la importancia de continuar trabajando e investigando en esta línea, así como la necesidad de implementar estas metodologías para comprobar, desde una perspectiva práctica, la hipótesis planteada.

\section{Bibliografía}

Asнока (2016): The guide to the seven key questions that all social entrepreneurs should ask themselves to have a clearer idea, develop their project and maximize their impact. Recuperado en https://docs.google.com/viewerng/viewer?url=https://www.ashoka.org/ sites/default/files/atoms/files/7qc-ashoka_switzerland-eng-web.pdf (fecha de último acceso: 28 de julio de 2017).

Austin, James; STEVenson, Howard; y Wei-Skillern, Jane (2006): «Social and commercial entrepreneurship: same, different or both?», Entrepreneurship: theory \& practice, 1 (30), 1-22.

BAtLle, Roser (2013): El aprendizaje-servicio en España: el contagio de una revolución pedagógica necesaria, Madrid, PPC.

- (2012): El aprendizaje-servicio como entrenamiento al emprendimiento social. Recuperado en http://roserbatlle.net/wp-content/uploads/2012/03/aps-como-entrenamientoal-emprendimiento-social.pdf (fecha de último acceso: 28 de julio de 2017).

Blanco, María Ascensión; Garín, Juan Alberto; y Rodríguez, Rosa María (2012). «Emprendimiento social: desarrollo de competencias y valores en el marco universitario», en Rodríguez Jiménez, Rosa María (Coord.): Educación en valores en el ámbito universitario. Propuestas y experiencias, Madrid, Narcea, 45-54.

Bravo Monge, Cris (2016): "Las escuelas de pensamiento del emprendimiento social», Tec empresarial, 10 (3), 19-28.

Comisión Europea (2016): Social enterprises and the social economy going forward. A call for action from the Commission Expert Group on Social Entrepreneurship. Recuperado en http://www.febea.org/sites/default/files/report_geces_14.october.2016.pdf (fecha de último acceso: 28 de julio de 2017).

- (2014): Entrepreneurship Education: a guide for educators, Bruselas, Unión Europea.

De la Calle, Mercedes (2016): «Aprendizaje basado en proyectos (ABP): posibilidades y perspectivas en ciencias sociales», Íber. Didáctica de las Ciencias Sociales, Geografía e Historia, 82, 7-12.

Deeley, Susan J. (2016): El aprendizaje-servicio en Educación Superior: teoría, práctica y perspectiva critica (traducido por Sara Alcina Zayas), Madrid, Narcea Ediciones.

Del Moral, Cristina y Sobrino, Diego (2016): "Aprendizaje basado en proyectos (ABP) en ciencias sociales». En Íber. Didáctica de las Ciencias Sociales, Geografía e Historia, 82, 4-6.

Del Real Álvarez, Hermógenes; Bueno Campos, Eduardo; y Fernández Horrillo, Pablo (2012): «Fomento del emprendimiento en la juventud: incubadoras, viveros de empresa y parques científicos», Revista de estudios de juventud, 99, 89-107.

Del Valle López, Ángela y Villa Fernández, Nuria (2008): «Visión crítica sobre el aprendizaje basado en problemas: ventajas y dificultades», en Escribano, Alicia y Del Valle, Ángela (Coords.). El aprendizaje basado en problemas. Una propuesta metodológica en Educación Superior, Madrid, Narcea, 133-150. 
Diego Rodríguez, Iván y Vega Serrano, José Antonio (2015): La educación para el emprendimiento en el sistema educativo español. Año 2015, Madrid, Ministerio de Educación, Cultura y Deporte.

Domingo, Joan (2008): «El aprendizaje cooperativo», Cuadernos de trabajo social, 21, 231-246.

Fernández March, Amparo (2006): «Metodologías activas para la formación de competencias", Educatio Siglo XXI, 24, 35-56.

Fundación Príncipe de Girona (2013): Aprender a emprender: cómo educar el talento emprendedor. Recuperado en http://es.fpdgi.org/upload/projecte/aprender-aemprenderesp.pdf (fecha de último acceso: 28 de julio de 2017).

Gallego García, Conchi (2011): "Hacia una sociedad donde todos seamos impulsores de cambios», Revista española del tercer sector, 17, 17-40.

Grimes, Richard (2015): «Problem-based learning and legal education -a case study in integrated experiential study», Revista de Docencia Universitaria, 13 (1), 361-375.

Guil Marchante, Concha y Montoya Sánchez, María Socorro (2015): «El emprendimiento social y el empleo de calidad", Lan harremanak, 32, 180-206.

Johnson, David W.; Johnson, Roger T. y Holubec, Edythe J. (1994): El aprendizaje cooperativo en el aula, Buenos Aires, Paidós.

Lucas Mangas, Susana y Martínez Odría, Arantzazu (2012): «La implantación y difusión del Aprendizaje-Servicio en el contexto educativo español. Retos de futuro de una metodología de enseñanza-aprendizaje para promover la innovación en la Educación Superior», Revista del Congrés Internacional de Docència Universitària i Innovació, $1,1-14$.

MAIR, Johanna y MARTí, Ignasi (2006): «Social entrepreneurship research: a source of explanation, prediction and delight", Journal of world business, 41, 36-44.

Manzanares Moya, María Asunción (2008): «Sobre el aprendizaje basado en problemas». En Escribano, Alicia y Del Valle, Ángela (Coords.). El aprendizaje basado en problemas. Una propuesta metodológica en Educación Superior, Madrid, Narcea, 17-26.

Marín, Aramis; Morua Ramírez, Juan; y Schmitt, Christophe (2012): «El emprendimiento social y el desarrollo de valores cooperativos», Projectics/Proyéctica/Projectique, $11-12,147-155$.

Marina, José Antonio (2010): "La competencia de emprender», Revista de Educación, 351, 49-71.

Martin, Roger L. y Osberg, Sally (2007): «Social entrepeneurship: the case for definition", Stanford Social Innovation Review, 5 (2), 28-39.

Melián Navarro, Amparo y Campos Climent, Vanessa (2010): «Emprendedurismo y Economía Social como mecanismos de inserción sociolaboral en tiempos de crisis», Revesco, 100, 43-67.

Moreira, Patricia y Urriolagoitia, Lourdes (2011): «El emprendimiento social», Revista española del tercer sector, 17, 17-40.

OCDE (2004): Promoting entrepreneurship and innovative SMEs in a global economy: towards a more responsible and inclusive globalization. Executive summary of the background reports (Spanish translation). Recuperado en https://www.oecd.org/cfe/ smes/31951224.pdf (fecha de último acceso: 28 de julio de 2017).

Pérez Álvarez, María del Pilar (2011): Innovación metodológica y Espacio Europeo de Educación Superior. Experiencias docentes en el ámbito del derecho, Madrid, Dykinson.

Prieto Navarro, Leonor (2007): El aprendizaje cooperativo, Madrid, PPC. 
Puig Rovira, Josep María; Batlle, Roser; Bosch, Carme; y Palos, Josep (2007): Aprendizaje servicio: educar para la ciudadanía, Barcelona, Octaedro.

Puig Rovira, Josep María (2011): «Por qué el aprendizaje-servicio crea humanidad?», Aula de innovación educativa, 203-204, 10-15.

Restrepo Gómez, Bernardo (2005): «Aprendizaje basado en problemas (ABP): una innovación didáctica para la enseñanza universitaria», Educación y Educadores, 8, 9-20.

Roberts, Dave y Woods, Christine (2005): "Changing the world on a shoestring: the concept of social entrepreneurship", University of Auckland Business Review, 7 (1), 45 51.

Rodríguez-Sandoval, Eduardo; Vargas-Solano, Édgar Mauricio; y Luna-Cortés, Janeth (2010): «Evaluación de la estrategia «aprendizaje basado en proyectos»», Educación y Educadores, 13 (1), 13-25.

Romero Medina, Agustín y García Sevilla, Julia (2008): «La elaboración de problemas ABP», en García Sevilla, Julia (Coord.). El aprendizaje basado en problemas en la enseñanza universitaria, Murcia, Editum, 37-53.

Salinas Ramos, Francisco y Osorio Bayter, Lourdes (2012): «Emprendimiento y Economía Social, oportunidades y efectos en una sociedad en transformación», CIRIECEspaña, Revista de Economía Política, Social y Cooperativa, 75, 129-151.

Santos Rego, Miguel Ángel; Sotelino Losada, Alexandre; y Lorenzo Moledo, María del Mar (2015): Aprendizaje-servicio y misión cívica de la universidad: una propuesta de desarrollo, Barcelona, Octaedro.

Sharan, Yael (2014): "Learning to cooperate for cooperative learning», Anales de Psicología, 30 (3), 802-807.

Uruñuela Nájera, Pedro María (2011): «Aprendizaje-servicio, una metodología para el desarrollo de la convivencia», Revista Amazônica, 6 (1), 35-61.

Vinuesa, María Pilar (2002): Construir los valores. Curriculum con aprendizaje cooperativo, Bilbao, Desclée.

Vizcarro, Carmen y JuÁrez, Elvira (2008): «¿Qué es y cómo funciona el aprendizaje basado en problemas?", en García SEvilla, Julia (Coord.). El aprendizaje basado en problemas en la enseñanza universitaria, Murcia, Editum, 17-36.

Zahra, Shaker A.; Gedajlovic, Erik; Neubaum, Donald O. y Shulman, Joel M. (2009): "A typology of social entrepreneurs: Motives, search processes and ethical challenges", Journal of business venturing, 24, 519-532. 